2. Hecht $\mathrm{S}$, Haig $\mathrm{C}$, Chase AM. The influence of light adaptation on subsequent dark adaptation of the eye. J Gen Physiol 1937; 20:831-50.

3. Lamb TD, Pugh EN Jr. Dark adaptation and the retinoid cycle of vision. Prog Retin Eye Res 2004;23:307-80.
4. Cameron AM, Mahroo OA, Lamb TD. Dark adaptation of human rod bipolar cells measured from the b-wave of the scotopic electroretinogram. J Physiol 2006;575:507-26.

DOI: $10.1056 /$ NEJMc1514294

\title{
Failure of Dual Antimicrobial Therapy in Treatment of Gonorrhea
}

TO THE EDITOR: Resistance to all antimicrobial agents has developed in some Neisseria gonorrhoeae strains. Dual antimicrobial therapy (ceftriaxone plus azithromycin) is a recommended first-line empirical treatment in many countries. ${ }^{1-3}$ We describe treatment failure with dual therapy in a patient with gonorrhea.

In December 2014, a heterosexual man presented to a sexual health clinic in the United Kingdom with a 2-week history of urogenital symptoms (Table 1). Ten days previously, he had returned from Japan, where his Japanese female partner had been treated for gonorrhea. $\mathrm{He}$ reported having no other recent sexual partners.

N. gonorrhoeae was detected in a urine specimen and pharyngeal swab on nucleic acid amplification testing (Abbott RealTime CT/NG assay) and in a culture of a urethral specimen. All N. gonorrhoeae-positive specimens on nucleic acid amplification testing were also confirmed as positive with the use of a duplex polymerasechain-reaction (PCR) assay targeting the porA pseudogene and opa genes. According to the local laboratory, testing with the disk-diffusion method showed that the $N$. gonorrhoeae strain was resistant to cefuroxime, ciprofloxacin, and tetracycline. The patient declined to undergo testing for syphilis and human immunodeficiency virus infection.

The patient received one dose of ceftriaxone intramuscularly at a dose of $500 \mathrm{mg}$ plus $1 \mathrm{~g}$ of azithromycin orally. ${ }^{3}$ At the test of cure on day 15 , a urine specimen was negative, but a pharyngeal swab remained positive for N. gonorrhoeae on the identical nucleic acid amplification test. The patient reported that he did not have sexual contact after treatment, and he did not return until day 79, when a pharyngeal swab was positive for N. gonorrhoeae on the nucleic acid amplification test.
On day 98, N. gonorrhoeae was detected in a pharyngeal sample on the nucleic acid amplification test and culture. The patient received one dose of ceftriaxone at a dose of $1 \mathrm{~g}$ intramuscularly plus azithromycin at a dose of $2 \mathrm{~g}$ orally. ${ }^{3}$ At the test of cure on day 112, the pharyngeal specimen was negative (according to the nucleic acid amplification test). Initial pretreatment specimens were unavailable for further analysis.

The N. gonorrhoeae species was verified with the use of the Phadebact Monoclonal GC Test and matrix-assisted laser desorption ionizationtime of flight mass spectrometry. Antimicrobial susceptibility testing with the use of Etest showed that the strain was resistant to ceftriaxone, azithromycin, cefixime, cefotaxime, penicillin, tetracycline, and ciprofloxacin, but it was susceptible to spectinomycin. Whole-genome sequencing of one isolate with the use of Illumina MiSeq (BioProject accession number PRJNA305360) and conventional sequencing identified N. gonorrhoeae multilocus sequence type ST1901 and a new N. gonorrhoeae multiantigen sequence type ST12133 in all specimens (the isolate and PCR specimens). Resistance determinants, ${ }^{1}$ mosaic penicillin-binding protein $2 \mathrm{X}$ (which decreases ceftriaxone target affinity), deletion of one adenine in the mtrR promoter (which increases MtrCDE efflux of ceftriaxone and azithromycin), and penB (which decreases PorB influx of ceftriaxone and azithromycin) were detected in all specimens.

The patient was considered to have treatment failure because the post-treatment isolate was resistant to ceftriaxone and azithromycin, all specimens contained resistance determinants and identical sequence types, and reinfection was deemed to be unlikely. The N. gonorrhoeae strain that caused the failure belonged to the identical $N$. gonorrhoeae multiantigen sequence 


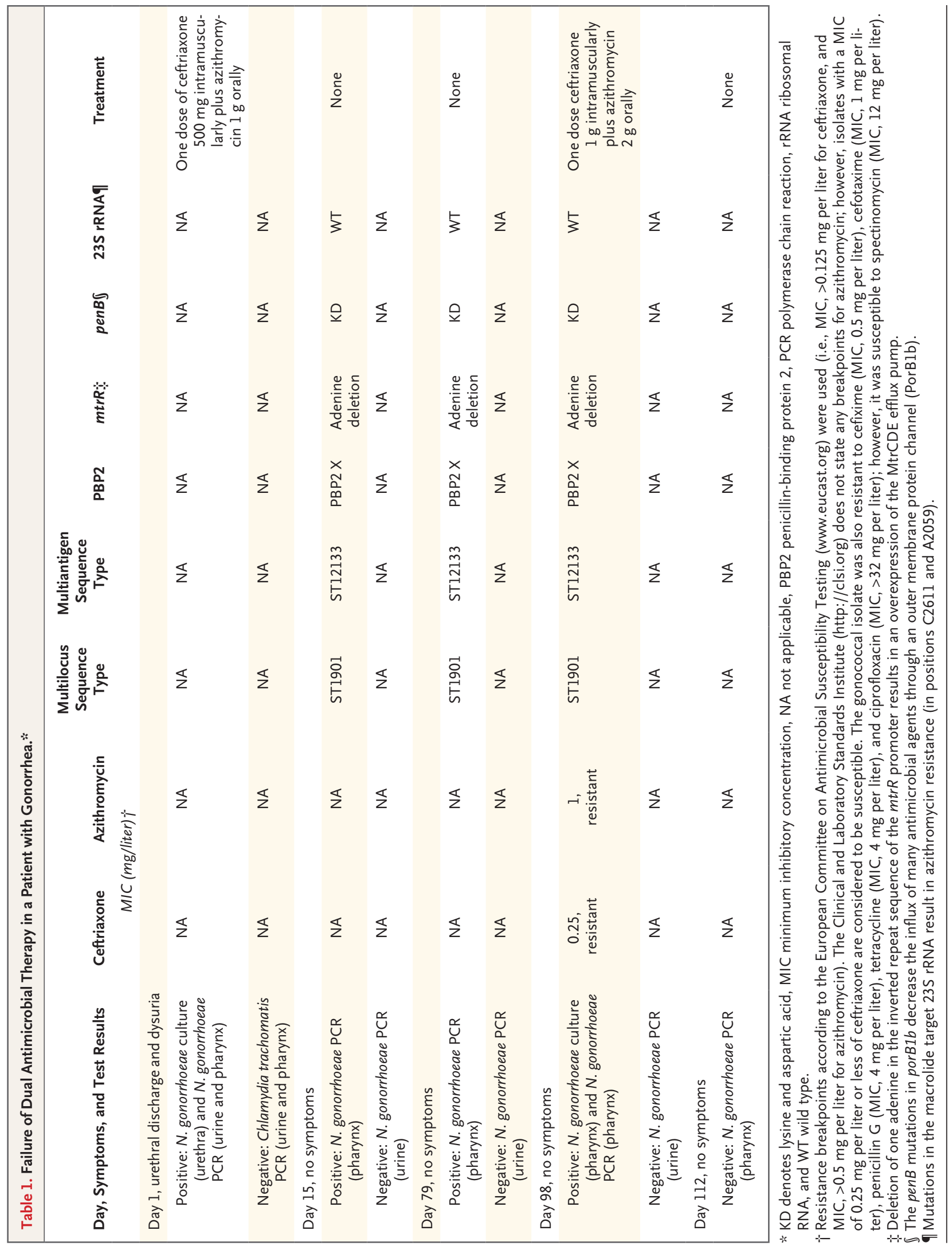

N ENGLJ MED 374;25 NEJM.ORG JUNE 23, 2016 
type genogroup as multilocus sequence type ST1901, N. gonorrhoeae multiantigen sequence type ST6800, which is spreading in Japan and is associated with decreased susceptibility to cephalosporins and azithromycin. ${ }^{4,5}$

In addition, the treatment failure reflected difficulties in treating pharyngeal gonorrhea as compared with urogenital gonorrhea. ${ }^{1,3}$ Pharyngeal gonorrhea is rare in heterosexual men. However, this patient reported no homosexual exposure; this highlights the need to test all potential sites of infection. A test of cure, partner notification and treatment, and effective antimicrobial stewardship and robust surveillance need to be considered so that gonorrhea may continue to be a treatable infection.

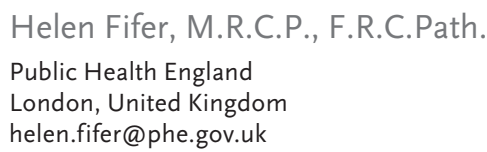

Usha Natarajan, M.R.C.P.I., F.R.C.P.

Lucy Jones, B.Sc.

Virgin Care

London, United Kingdom

Sarah Alexander, Ph.D.

Gwenda Hughes, Ph.D., F.F.P.H.

Public Health England

London, United Kingdom

Daniel Golparian, M.Sc.

Magnus Unemo, Ph.D.

Örebro University

Örebro, Sweden

Disclosure forms provided by the authors are available with the full text of this letter at NEJM.org.

1. Unemo M, Shafer WM. Antimicrobial resistance in Neisseria gonorrhoeae in the 21st century: past, evolution, and future. Clin Microbiol Rev 2014;27:587-613.

2. Bolan GA, Sparling PF, Wasserheit JN. The emerging threat of untreatable gonococcal infection. N Engl J Med 2012;366: 485-7.

3. Unemo M. Current and future antimicrobial treatment of gonorrhoea - the rapidly evolving Neisseria gonorrhoeae continues to challenge. BMC Infect Dis 2015;15:364.

4. Shimuta K, Unemo M, Nakayama S, et al. Antimicrobial resistance and molecular typing of Neisseria gonorrhoeae isolates in Kyoto and Osaka, Japan, 2010 to 2012: intensified surveillance after identification of the first strain (H041) with highlevel ceftriaxone resistance. Antimicrob Agents Chemother 2013;57:5225-32.

5. Shigemura K, Osawa K, Miura M, et al. Azithromycin resistance and its mechanism in Neisseria gonorrhoeae strains in Hyogo, Japan. Antimicrob Agents Chemother 2015;59: 2695-9.

DOI: $10.1056 /$ NEJMc1512757

Correspondence Copyright @ 2016 Massachusetts Medical Society.

\section{INSTRUCTIONS FOR LETTERS TO THE EDITOR}

Letters to the Editor are considered for publication, subject to editing and abridgment, provided they do not contain material that has been submitted or published elsewhere. Please note the following:

- Letters in reference to a Journal article must not exceed 175 words (excluding references) and must be received within 3 weeks after publication of the article.

- Letters not related to a Journal article must not exceed 400 words.

- A letter can have no more than five references and one figure or table.

- A letter can be signed by no more than three authors.

- Financial associations or other possible conflicts of interest must be disclosed. Disclosures will be published with the letters. (For authors of Journal articles who are responding to letters, we will only publish new relevant relationships that have developed since publication of the article.)

- Include your full mailing address, telephone number, fax number, and e-mail address with your letter.

- All letters must be submitted at authors. NEJM.org.

Letters that do not adhere to these instructions will not be considered. We will notify you when we have made a decision about possible publication. Letters regarding a recent Journal article may be shared with the authors of that article. We are unable to provide prepublication proofs. Submission of a letter constitutes permission for the Massachusetts Medical Society, its licensees, and its assignees to use it in the Journal's various print and electronic publications and in collections, revisions, and any other form or medium.

THE JOURNAL'S WEB AND E-MAIL ADDRESSES

To submit a letter to the Editor: authors.NEJM.org

For information about the status of a submitted manuscript: authors.NEJM.org

To submit a meeting notice: meetingnotices@NEJM.org

The Journal's web pages: NEJM.org 